\title{
Architecture to Serve Disabled and Elderly
}

\author{
Miriam Buonamente and Magnus Johnsson
}

\begin{abstract}
We propose an architecture (discussed in the context of a dressing and cleaning application for impaired and elderly persons) that combines a cognitive framework that generates motor commands with the MOSAIC architecture which selects the right motor command according to the proper context. The ambition is to have robots able to understand humans intentions (dressing or cleaning intentions), to learn new tasks only by observing humans, and to represent the world around it by using conceptual spaces. The cognitive framework implements the learning by demonstration paradigm and solves the related problem to map the observed movement into the robot motor system. Such framework is assumed to work with two operative modalities: observation and imitation. During the observation the robot identifies the main actions and the properties of the involved objects; hence it classifies, organizes and labels them. This is done to create a repertoire of actions and to represent the world around. During the imitation the robot selects the proper rules to reproduce the observed movement and generate the proper motor commands. The end goal is to connect the generated motor commands to the operative context (dressing or cleaning). The MOSAIC architecture is the suitable solution for this problem. MOSAIC is made of multiple couplings of predictors, which predict system motor behaviour, and controllers which properly select the motor command depending on the context. The proposed architecture presents one controller for each context and each controller is paired with one predictor. The motor commands generated by the framework are sent to the predictor, whose estimates are then compared with the current sensory feedback and the difference between them generates a prediction error. The smaller the prediction error, the more likely the context. Once the right prediction is identified, the paired controller is selected and used.
\end{abstract}

Miriam Buonamente

Department of Chemical, Management, Computer, Mechanical Engineering of the University of Palermo, Palermo, Italy e-mail: miriambuonamente@gmail.com

Magnus Johnsson

Lund University Cognitive Science, Kungshuset, Lundagård, 22222 Lund, Sweden, e-mail: magnus@magnusjohnsson.se 